\title{
Upscaling UAV-borne high resolution vegetation index to satellite resolutions over a vineyard
}

\author{
Y. Wang ${ }^{1}$, D. Ryu ${ }^{1}$, S. Park ${ }^{1}$, S. Fuentes ${ }^{2}$ and M. O'Connell ${ }^{3}$ \\ ${ }^{1}$ Department of Infrastructure Engineering, The University of Melbourne, Parkville, Victoria, Australia \\ 2 School of Agriculture and Food, Faculty of Veterinary and Agricultural Sciences, The University of \\ Melbourne, Parkville, Victoria, Australia \\ ${ }^{3}$ Department of Economic Development, Jobs, Transport and Resources, Tatura, Victoria, Australia
}

Email: wang.y@unimelb.edu.au

\begin{abstract}
Vegetation indices retrieved by optical satellite sensors provide important information related to the vigour, biomass and growth of plants. Homogeneous biophysical state within a satellite footprint is often assumed to make the spectral vegetation indices a reliable predictor of the vegetation condition. However, the image pixel resolution for the most widely used optical satellites, such as Landsat (30 m by $30 \mathrm{~m}$ ) and Moderate Resolution Imaging Spectroradiometer (MODIS, $250 \mathrm{~m}$ by $250 \mathrm{~m} \sim 500 \mathrm{~m}$ by $500 \mathrm{~m}$ ), includes vegetation canopies with varying degree of heterogeneity within single pixels. This pixellevel heterogeneity makes it difficult to predict individual plant-level vegetation biomass and vigour from satellite imagery. In particular, the structured nature of vineyard fields comprising predominantly of bare soil and grapevines pose challenges when estimating the vine-level biomass and vigour from the coarseresolution satellite images, that present signals averaged over vine canopies and non-canopies (e.g., soil). In this study, we examine how the normalized difference vegetation index (NDVI) varies with aggregating pixel scales from sub-5-cm to $30-\mathrm{m}$ and $250-\mathrm{m}$ resolutions using very-high-resolution images collected by a multispectral imaging sensor on-board an unmanned aerial vehicle (UAV). The aggregated 30-m and 250-m scale NDVI values were compared with Landsat $8(30 \mathrm{~m})$ and MODIS (250 m) products collected from a commercial vineyard in Victoria, Australia within a narrow time window (25 hours). Results showed that when upscaling the high-resolution NDVI values (by aggregating input multispectral bands for NDVI) to coarser resolutions in highly structured vineyard fields, NDVI decreased initially up to the field heterogeneity scale (spacing between vine rows in this study site) and then levelled off beyond the scale. Furthermore, the difference between NDVIs at Landsat 8 pixels was mainly caused by the areal fractions of canopy and soil pixels and the NDVI values of the soil rows.
\end{abstract}

Keywords: Normalized Difference Vegetation Index (NDVI), Unmanned Aerial Vehicle (UAV), pixel aggregation, upscaling imagery 


\section{INTRODUCTION}

Accurate monitoring of vegetation vigour and biomass over large fields is an important component of precision agriculture. Over large agricultural fields, satellite remote sensing can provide a suitable coverage with a moderate revisit time. The spatial and temporal coverage of satellite observations can be enhanced by combining multiple products that have different spatial resolutions and instrument features. However, it is important to understand inherent discrepancy between different products originating from the spatial, spectral and other instrument features since they can influence the scientific outputs, from different satellites or other remote sensing platforms. For example, Abuzar et al (2014) demonstrated difference in NDVI values from different platforms over horticulture crops in south-eastern Australia.

Multispectral sensors on-board the Unmanned Aerial Vehicles (UAVs) have been attracting significant attention as a versatile platform to capture high-resolution imagery in a fast and economic way to support agricultural management decisions. Utility of UAV-borne sensing is remarkable particularly for small scale applications (Grenzdorffer et al., 2008). Also, the high-resolution imagery obtained by UAVs provides a unique opportunity to examine the effect of aggregating pixel scales, from centimetres to the scales of popular optical satellites such as Landsat $(30 \mathrm{~m}$ by $30 \mathrm{~m}$ ) and MODIS ( $250 \mathrm{~m}$ by $250 \mathrm{~m} \sim 500 \mathrm{~m}$ by $500 \mathrm{~m}$ ).

The main aim of this study is to address how vegetation indices vary across different pixel scales among canopy and non-canopy, respectively. For this purpose, NDVI values retrieved from multiple remote sensing platforms at different scales, from sub-5-cm (UAV) to 250-m (MODIS) are compared. In the course of the multi-scale comparisons, following questions are aimed to be addressed: what is the influence of changing pixel scales on NDVI?; what are the main factors to be considered when estimating sub-grid-scale (individual plant level) NDVI from coarser scales at which heterogeneous land cover types of structured horticultural landscapes are lumped? To achieve the study objectives, this paper employs three representative scales imagery applying NDVI in a vineyard, including the imagery captured from UAV (eBee, senseFly, Ltd.), Landsat 8 and MODIS on-board the Aqua satellite for comparison.

\section{MATERIALS AND METHODOLOGY}

\subsection{Study area}

The study area is located at the Curly Flat Vineyard $\left(37^{0} 17^{\prime} 40^{\prime \prime} \mathrm{S}, 144^{0} 42^{\prime} 24^{\prime \prime} \mathrm{E}\right)$ with average elevation of 560 metres in the Macedon Ranges of Victoria, Australia. It covers approximately 14 hectares with progressively high vegetation coverage since first plantings in 1992, including 70\% Pinot Noir, 25\% Chardonnay and 5\% Pinot Gris. All vines are grafted with two main scaffold branches known as Lyre Trellis with an inter-plant space of $0.9 \mathrm{~m}$. Seven highly structured vineyard fields numbered from 1 to 7 in Figure 1 with a row and plant space as $3 \mathrm{~m}$ and $1.8 \mathrm{~m}$, respectively, provided an ideal study area to conduct upscaling analysis over fields with a large contrast among different vegetation fractions.

\subsection{Data Acquisition}

Three different scales of cloud-free imagery were obtained

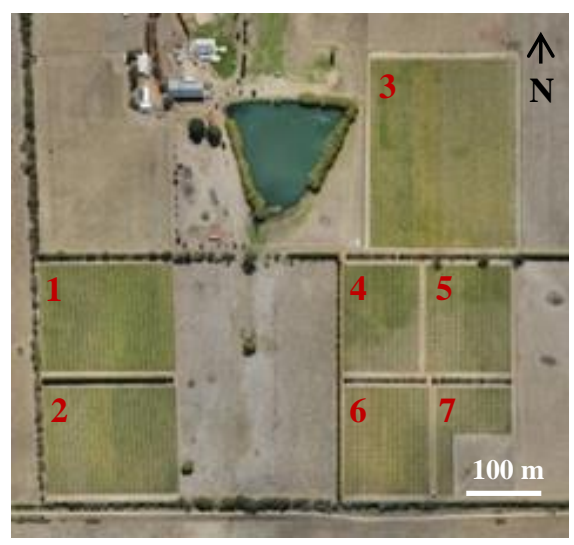

Figure 1. Study area of Curly Flat Vineyard on March 30, 2015 (mosaic image from eBee)

from UAV, Landsat and MODIS to map NDVI of the study area between the $29^{\text {th }}$ and $30^{\text {th }}$ of March 2015 , as described in Table 1.

Table 1. Attributes of different sensors used to derive NDVI

\begin{tabular}{|c|c|c|c|}
\hline Sensor & UAV (eBee) & LANDSAT 8 Level 2 & $\begin{array}{c}\text { AQUA MODIS Vegetation } \\
\text { Indices }\end{array}$ \\
\hline Date & 30 March 2015 & 29 March 2015 & 30 March 2015 \\
\hline Data Source & Field Survey & USGS & USGS \\
\hline Captured Time (Local time) & $11: 30 \mathrm{am}$ & $12: 30 \mathrm{pm}$ & $1: 30 \mathrm{pm}$ \\
\hline Red/NIR Band Wavelength $(\boldsymbol{\mu m})$ & $0.62 \pm 0.05 / 0.83 \pm 0.04$ & $0.65 \pm 0.02 / 0.87 \pm 0.02$ & $0.64 \pm 0.02 / 0.86 \pm 0.02$ \\
\hline Ground Spatial Resolution & $4 \mathrm{~cm}$ & $30 \mathrm{~m}$ & $250 \mathrm{~m}$ \\
\hline
\end{tabular}




\subsection{Methods}

Calculation of Vegetation Indices (VI) is a direct way to quantify the density of plant growth. In general, multiple spectral bands are combined in producing VIs to represent vegetation vigour and biomass. In fact, the reflectance of plants in the near-infrared (NIR) range is much higher than that in the visible band, especially the red band. Therefore, the contrast between responses of NIR and red band can be considered as a sensitive measurement for green leaves (Solano et al., 2010). In this study, the most commonly used NDVI to produce spectral VI to separate green leaves from the bare soil and water bodies to minimize topographic effects was implemented (Silleos et al., 2006).

In order to reduce the impact from difference between sensors and to improve the accuracy, it is necessary to conduct radiometric calibration and geometric rectification before

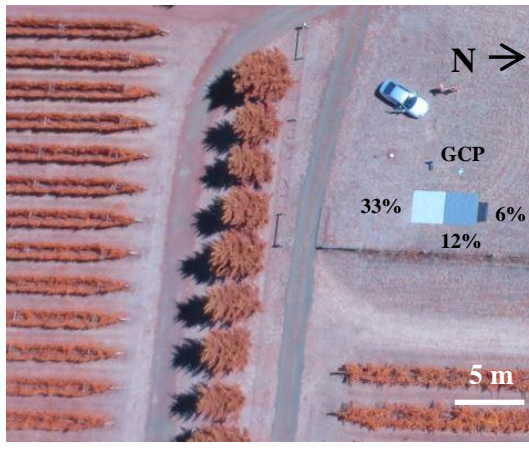

Figure 2. False color UAV imagery captured GCPs and calibration targets placed between Field 3 and 4 calculating the NDVI for each image to correct radiometric errors and geometric distortion. A total of 280 high-resolution UAV images over the Curly Flat Vineyard were georeferenced with 16 ground control points (GCPs). An empirical linear radiometric calibration was performed using known reflectance values over three artificial calibration targets, placed near the centre of the Vineyard as shown in Figure 2. The polyester target fabric represented three different constant reflectance values of $33 \%, 12 \%$ and $6 \%$, respectively. The digital number (DN) was converted to calibrate reflectance by the grey scale calibration using the linearity of reflectance for NIR and red bands. The NDVI is a normalized ratio of the surface reflectance of NIR and red bands, which can separate green vegetation from other surfaces, shown in Equation (1).

$$
N D V I=\frac{\rho N I R-\rho R}{\rho N I R+\rho R}
$$

The calculated NDVI value ranges from -1 to +1 . The value close to 1 is related to the highest possible density of green vegetation, while the value for bare soil, water bodies and clouds are near 0 (Weier and Herring, 2009). This study distinguished the canopy and non-canopy (e.g., soil background) pixels based on the NDVI distribution with a threshold value of 0.52. Additionally, the threshold NDVI value of Landsat pixel was 0.7 for high vegetation pixels, 0.6 for intermediate vegetation pixels and less than 0.5 for low vegetation pixels. After calculating individual NDVI values, it was assessed how the NDVI varied among different-scale platforms by conducting resampling from small scales to large scales in gradual levels. When upscaling UAV imagery to the Landsat scale, we resampled the reflectance of each input band (red and NIR bands) in 6 increments of $10 \mathrm{~cm}, 50 \mathrm{~cm}, 1 \mathrm{~m}, 3 \mathrm{~m}, 10 \mathrm{~m}$ and $30 \mathrm{~m}$, then the resampled reflectance for red and NIR bands was used to calculate NDVI values in each corresponding scale. The same method was applied to aggregate pixels from the Landsat scale to the MODIS scale in three increments $100 \mathrm{~m}, 200 \mathrm{~m}$ and $250 \mathrm{~m}$. The upscaling of spatial resolution involved the linear aggregation of high-resolution imagery into coarser resolution with fewer pixels, which naturally smears out details of the original data (Jones and Vaughan, 2010). In this work, ENVI 5.3 (Harris, Inc.), ArcGIS 10.3 (Esri, Inc.) and PhotoScan (Agisoft, LLC.) were used to process, analyse and integrate the images.

\section{RESULTS AND DISCUSSION}

\subsection{NDVI maps from different platforms}

The NDVI maps derived from UAV, Landsat 8 and MODIS of the entire study area are shown in Figure 3 in an NDVI range of 0 to 1 . As presented in the NDVI maps of three different scales, UAV and Landsat preserved a better contrast between water (close to 0), soil (approximately 0.4) and canopy (close to 1). On the other hand, the coarser-scale MODIS NDVI provides only general information of vegetation existence with NDVI values varying between 0.3 and 0.6. In general, the NDVI values are intermediate between the values of soil and vegetation canopy, and exhibits lower values over the northwest and southern parts of the study area where the bare soil fraction is dominant. However, the NDVI map of the Landsat scale shows the boundary of each subfield more clearly than MODIS scale, making it possible to distinguish between vineyard fields (green), bare soil (yellow) and the open water body (brown). The Landsat data shows the highest NDVI over Field 1 (field number as represented in Figure 1) among all the seven fields. Additionally, compared with the coarser NDVI maps, the finest NDVI from UAV provides a good representation of the 
details in the highly structured grapevine fields. For example, in Field 3 as shown in Figure 4, the individual highly structured vine rows and even individual vines can be recognised from the UAV scale. From the Landsat scale, some intra field spatial variation in NDVI can be visualized, where it was higher in the west compared to the east in Figure 4 (a). Greater detail in spatial variability of NDVI was recognized from the UAV scale, where the high NDVI values were clustered in the west than the east. More specifically, the central part of the Field 3 in the white dash-line boundary, the NDVI showed slightly higher values than its adjacent pixels in the same column in Figure 4 (b). From the UAV scales, higher fraction of canopy cluster was found to the left in the white dotted box, which matched the changes in the Landsat pixel. However, it is only possible to get a very rough idea of the mean NDVI in a large area for MODIS compared to the smaller scales.
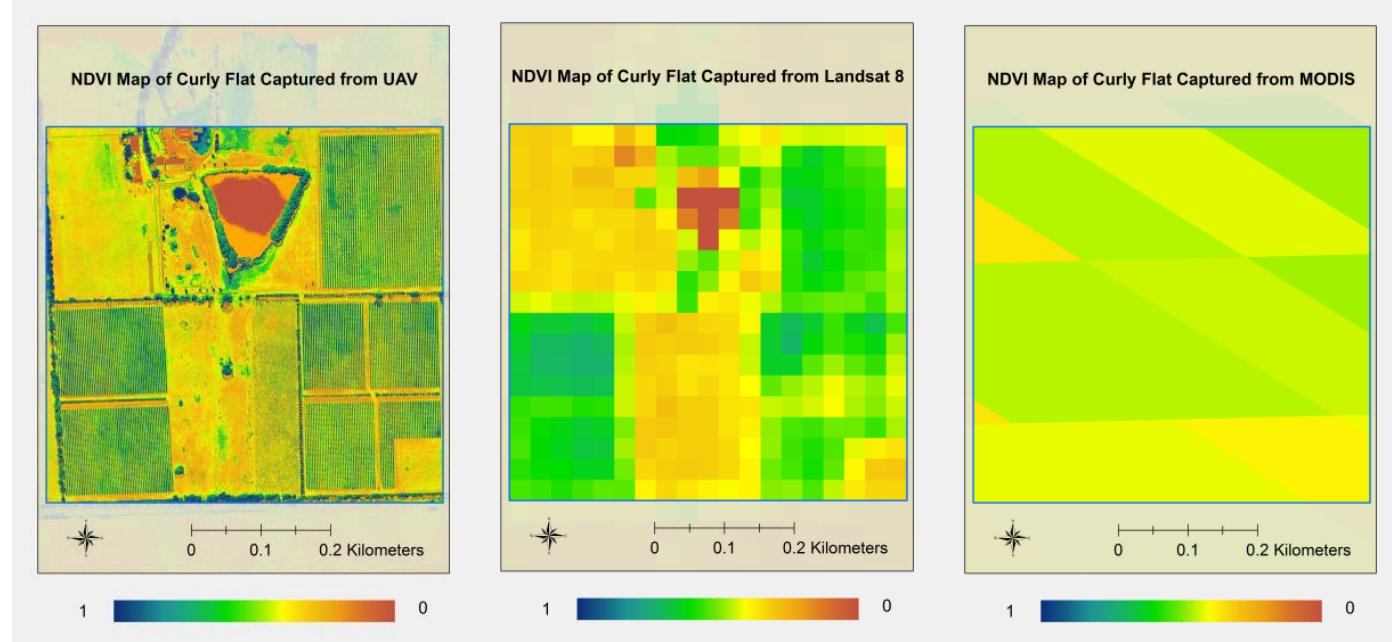

Figure 3. NDVI maps from different platforms in the study area

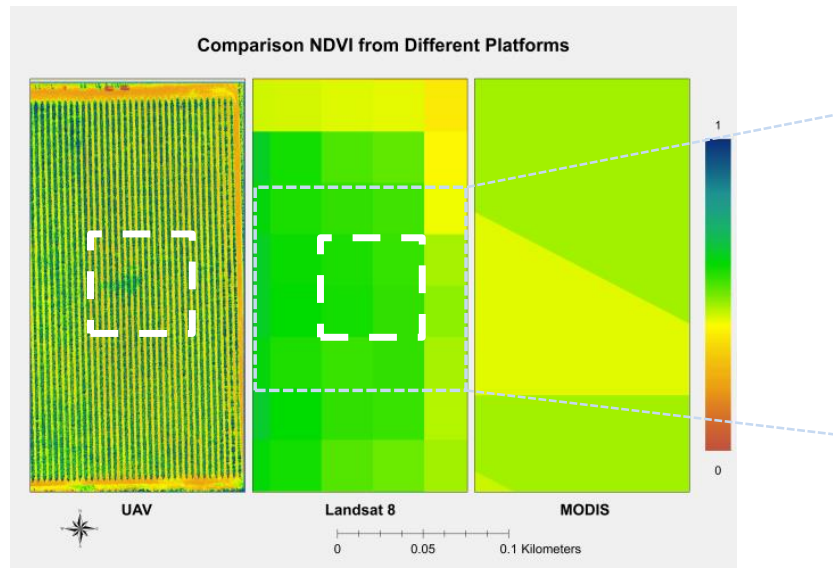

(a)

Figure 4. (a) Comparison NDVI map in Field 3 and (b) NDVI values in a specific area from Landsat platform

\subsection{Upscaling from UAV to Landsat 8 resolution}

After comparing the NDVI values from three different platforms at the pixel scales, $4 \mathrm{~cm}, 30 \mathrm{~m}$ and $250 \mathrm{~m}$, we conducted a systematic aggregation of the UAV imagery to examine how the NDVI values vary across the scales. Analysis of NDVI changes with reducing resolution (pixel aggregation) was applied to six Landsat 8 pixels randomly chosen in three intervals of NDVI values ( 2 pixels for each interval as shown in Figure 5): $\mathrm{H}$ represents high vegetation pixels (NDVI $>0.7), \mathrm{M}$ denotes intermediate vegetation pixels $(\mathrm{NDVI} \approx 0.6)$ and $\mathrm{L}$ stands for low vegetation pixels (NDVI < 0.5 ) of Landsat 8. 


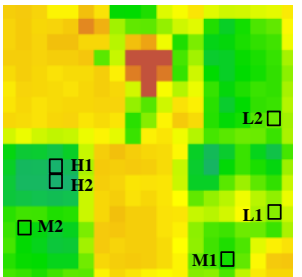

(a) Landsat 8 NDVI

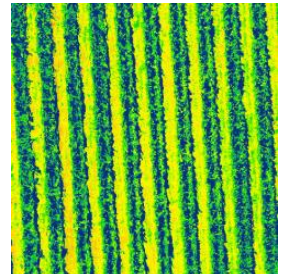

(b) H1 (High NDVI grid)

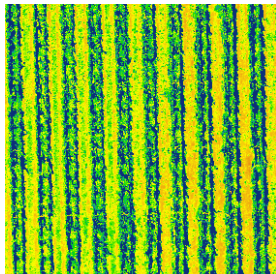

(c) M1 (Intermediate NDVI grid)

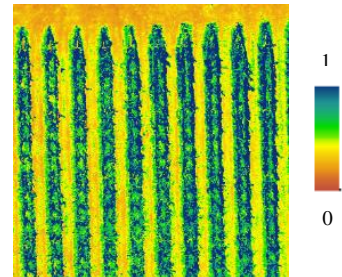

(d) L1 (Low NDVI grid)

Figure 5. (a) Distribution of random selected pixels in three different vegetation levels in the Landsat 8 NDVI (high, intermediate and low); (b) an example of high NDVI grid (30 m by $30 \mathrm{~m}$ ) (H1); (c) intermediate NDVI grid (M1); (d) low NDVI grid (L1)

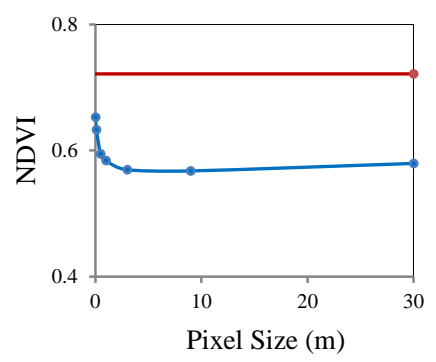

(a) High Vegetation Pixels

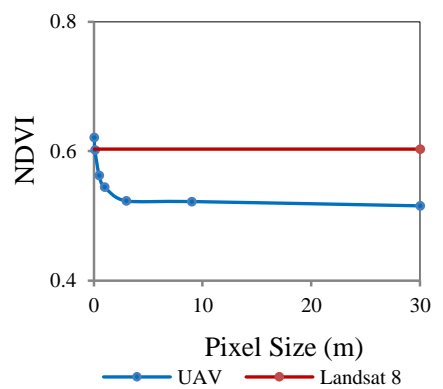

(b) Intermediate Vegetation Pixels

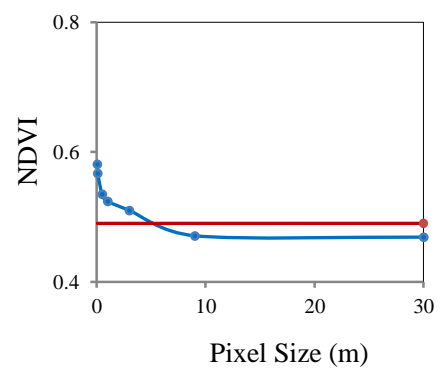

(c) Low Vegetation Pixels

Figure 6. Upscaling UAV NDVI in different mean NDVI level sampling grids

\section{High NDVI Grid}

The high-NDVI pixels of Landsat 8, two adjacent pixels $\mathrm{H} 1$ and $\mathrm{H} 2$, both had NDVI of 0.72 were located in Field 1. As shown in the UAV-borne NDVI maps of the sample pixels in Figures 5 (b) - (d), the high-NDVI is mainly attributed to the denser canopies and soil rows of the block. The fractions of soil and canopy pixels were 0.60 and 0.40 , and the corresponding averaged NDVI values of them were 0.47 and 0.94 , respectively. The mean NDVI for the highest resolution of the UAV-borne imagery over $\mathrm{H}$ pixels was lower than the Landsat 8 NDVI by a small margin of 0.06 . However, the UAV-borne NDVI declined systematically with the pixel aggregation $(0.04 \mathrm{~m}, 0.1 \mathrm{~m}, 0.5 \mathrm{~m}, 1 \mathrm{~m}, 3 \mathrm{~m}, 10 \mathrm{~m}$ and $30 \mathrm{~m}$ as shown in Figure 6 (a)). The difference between the UAV-borne and Landsat 8 NDVI values increased at the scales from $0.04 \mathrm{~m}$ to $1 \mathrm{~m}$ then levelled off from $3 \mathrm{~m}$, resulting in the final difference in NDVI of 0.14 at 30-m resolution.

\section{Intermediate NDVI Grid}

The intermediate NDVI pixels, M1 (0.58 for the Landsat 8 pixel) and M2 (0.60), were chosen from the Fields 2 and 6, respectively. The averaged NDVI values of the UAV data in the intermediate grid (M) as 0.94 for canopy and 0.44 for soil were similar to those from high grid as shown in Table 2 . However, the pixelaverage NDVI values were lower for $\mathrm{M}$ due to the smaller canopy fractions as 0.32 (smaller and sparser canopy coverage). The mean UAV NDVI values are close to the Landsat 8 NDVI when the high-resolution $(0.4 \mathrm{~m}$ and $0.1 \mathrm{~m})$ NDVI was used. But the mean NDVI again declines up to the $3-\mathrm{m}$ resolution then levels off to the minimum value (see Figure 6 (b)). The mean NDVI of UAV data, however, showed values slightly higher than the Landsat $8 \mathrm{NDVI}$ and the converged NDVI at over $3 \mathrm{~m}$ scales presented smaller difference than the high-NDVI samples.

\section{Low NDVI Grid}

For the low NDVI pixels of L1 and L2, located on the edge of Fields 3 and 7, vegetation was represented less strongly than higher vegetation pixels, since large bare soil surfaces (74\% coverage) were included on the edge of each L1 and L2 grid (Figure 5 (d)). The UAV NDVI showed two points of inflections when upscaling NDVI at approximately $3 \mathrm{~m}$ and $10 \mathrm{~m}$ in pixel size (Figure 6 (c)), indicating two scales of heterogeneity: (1) the size of inter-row distance $(3 \mathrm{~m})$, which is between a vine row and a soil row; (2) the size of the bare soil area at the northern edge of the low vegetation grids. Since the first point of inflection (at 
$3 \mathrm{~m}$ ) is caused by the size of inter-row distance, high and intermediate UAV NDVIs also represented the identical characteristics of inflection point at $3 \mathrm{~m}$ pixel size and turned to be stable (Figure 6 (a) and 6 (b)). The mean UAV NDVI (L) was closer to the Landsat NDVI compared to higher vegetation pixels (H and $\mathrm{M})$.

\section{Overall of upscaled UAV NDVIs}

For upscaling from UAV to Landsat 8, the averaged UAV NDVI in the Landsat pixel grid (30 $\mathrm{m}$ by $30 \mathrm{~m}$ ) decreased slightly until $3 \mathrm{~m}$ and then kept constant until $30 \mathrm{~m}$ scale. Furthermore, the difference of NDVI values between UAV and Landsat was getting closer from higher to lower vegetation pixels. In other words, the Landsat NDVI kept dropping until intersecting with UAV from higher to lower vegetation pixels when increasing the pixel size from $0.04 \mathrm{~m}$ to $30 \mathrm{~m}$. As shown in Table 2, the NDVIs of averaged canopy were similar around 0.94 among high, intermediate and low vegetation pixels except for Landsat 8 pixel L2 (0.92), but the NDVIs of averaged soil were more variable among Landsat 8 pixels. The latter shows that the differences among the NDVIs were mainly affected by the relative fractions of soil and canopy areas in this highly structured vine field. The pattern in Figure 7, which was located in the Fields 4 and 5, represents the NDVI distribution from the original UAV scale, upscaled UAV to $3 \mathrm{~m}$, upscaled UAV to $30 \mathrm{~m}$ and original Landsat scale $(30 \mathrm{~m})$. The fractions of high NDVI and low NDVI were significant shown in this upscaling UAV map. For example, the Fields 4 and 5 (Figure 7) includes an open area where bare soil surface and low densities of vegetation exist in the boundary between two fields and the bottom of the fields. When upscaling, NDVIs have merged and the details of NDVI have lost, however, the major pattern of the NDVIs were still remained. In the result, the upscaled UAV NDVI showed similar values and spatial distributions to the Landsat NDVI, showing the difference of 0.12 .

Table 2. Statistics of individual NDVI within selected Landsat pixels

\begin{tabular}{|c|c|c|c|c|}
\hline PDI & $\begin{array}{c}\text { Averaged } \\
\text { Soil }\end{array}$ & $\begin{array}{c}\text { Averaged } \\
\text { Canopy }\end{array}$ & $\begin{array}{c}\text { Averaged } \\
\text { UAV }\end{array}$ & $\begin{array}{c}\text { Landsat } \\
\mathbf{8}\end{array}$ \\
\hline H1 & 0.4706 & 0.9384 & 0.6526 & 0.7215 \\
\hline H2 & 0.4703 & 0.9413 & 0.6593 & 0.7268 \\
\hline M1 & 0.4456 & 0.9388 & 0.6019 & 0.5834 \\
\hline M2 & 0.4397 & 0.9402 & 0.6213 & 0.6030 \\
\hline L1 & 0.4078 & 0.9349 & 0.5812 & 0.4900 \\
\hline L2 & 0.4084 & 0.9190 & 0.5415 & 0.4591 \\
\hline
\end{tabular}

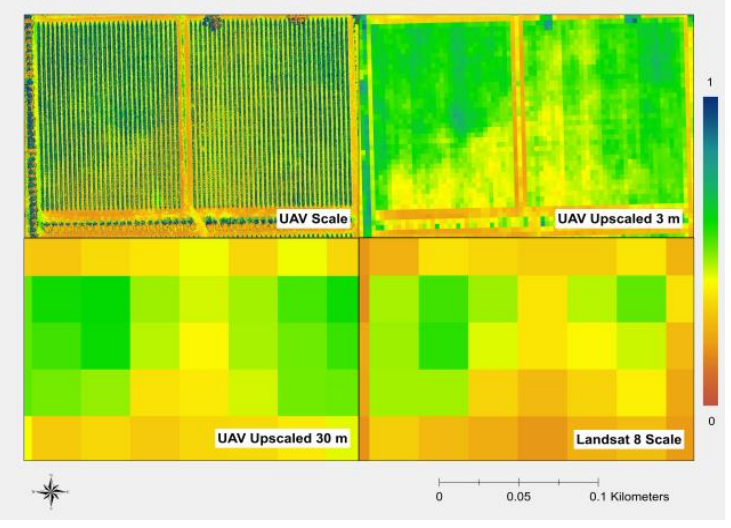

Figure 7. NDVI scale comparisons from UAV to Landsat 8 in Fields 4 and 5

\subsection{Upscaling from UAV and Landsat 8 to MODIS scale}

When upscaling from UAV and Landsat scales to MODIS scale, a centred pixel from MODIS was chosen as the optimal one to cover the vines as much as possible. The pixel covered most of the Fields 4 and 5 and some of the Fields 3 and 7, as highlighted in red parallelogram MODIS pixel with UAV NDVI map underneath in Figure 8. In total, $23 \%$ of the pixels were covered with the highly structured vine canopy from the UAV scale. The averaged NDVIs were 0.93 and 0.37 for canopy and soil in the selected MODIS pixel size. And $0.49,0.47$ and 0.44 were the averaged NDVI for UAV, Landsat and MODIS, respectively.

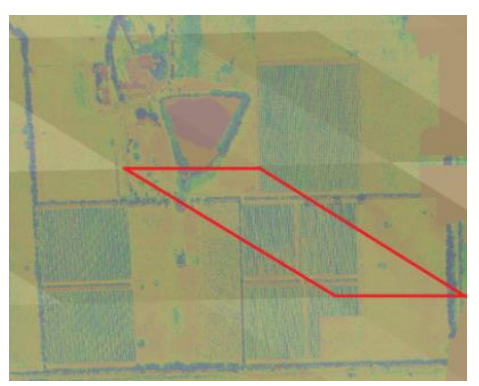

Figure 8. Location of the selected MODIS pixel

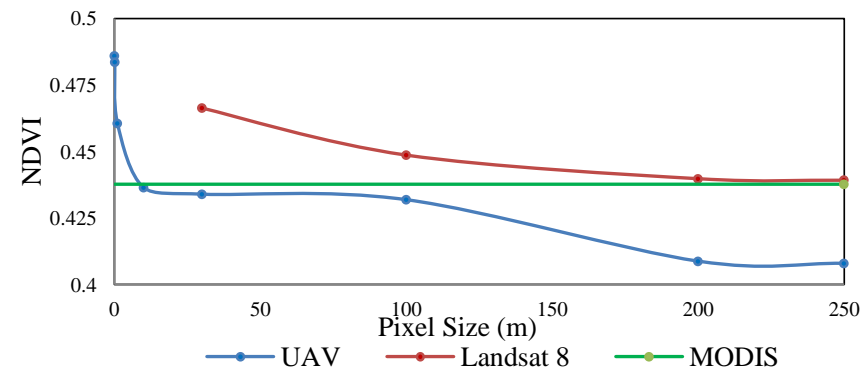

Figure 9. Averaged NDVI comparison within a MODIS pixel 
Wang et al., Upscaling UAV-borne high resolution vegetation index to satellite resolutions over a vineyard

For resampling pixel size from the UAV scale $(0.04 \mathrm{~m})$ to MODIS scale $(250 \mathrm{~m})$, it is possible to separate the upscaling into two stages with the demarcation point at $100 \mathrm{~m}$ in Figure 9 . The NDVI dropped to 0.44 in 10 $\mathrm{m}$, from $10 \mathrm{~m}$ to $100 \mathrm{~m}$ it decreased 0.01 in a slight fluctuation, and then fell to $0.41 \mathrm{in} 200 \mathrm{~m}$ with a steady value to the end. Within $100 \mathrm{~m}$, the fraction between the soil and canopy seemed to be stable in Field 4 and 5 . A significant change appeared when upscaling to $200 \mathrm{~m}$, at which the soil fraction increased significantly. Meanwhile, the Landsat NDVI declined gently and smoothly to meet MODIS when upscaling to MODIS scale. From $30 \mathrm{~m}$ to $250 \mathrm{~m}$, Landsat had the highest NDVI followed by MODIS and then the UAV.

\section{CONCLUSIONS}

This study used the UAV-borne high-resolution imagery to identify how NDVI varies when upscaling to coarse-resolution satellite pixels over a high structured commercial vineyard. In terms of MODIS resolution $(250 \mathrm{~m})$, it was not possible to distinguish the grapevine canopy, bare soil and other features within each subfield. For Landsat resolution $(30 \mathrm{~m})$, the details of the single canopy or vine-level structure were not detectable. It was found that the averaged NDVI from UAV declined gradually to a steady value when upscaling from the original resolution $(0.04 \mathrm{~m})$ to Landsat resolution $(30 \mathrm{~m})$. Meanwhile, the gap between the averaged UAV NDVI and Landsat NDVI reduced from higher to lower vegetation pixels during upscaling resample. For upscaling from Landsat to MODIS scale, the same tendency was showed with the NDVI that dropped at the start and then kept invariant. While from UAV to MODIS scale, the NDVI started falling to a constant value to reach the MODIS NDVI at $10 \mathrm{~m}$, and from $200 \mathrm{~m}$ it decreased dramatically again to a stable value. The possible reason for these changes might be associated to the resampled pixel location in an area with different fraction of features, a large fraction of soil located beyond the field with high vine structure.

Overall, the averaged canopy NDVIs extracted from UAV were similar across the H, M and L sampling grids with different NDVI levels. However, NDVIs averaged over soil rows varied between the sampling grids, and L pixels contained larger fraction of soil pixels with lower soil NDVIs. Therefore, it is concluded that the difference between the upscaled NDVIs was mainly influenced by the soil NDVI and the areal fractions of canopy to soil pixels. This study presented different spatial resolutions of NDVI upscaled from subcentimetre resolution and examined the comparisons between the upscaled UAV NDVI and the coarse scale NDVIs from the Landsat and MODIS. As a future work, further research on various vegetation fields with different types of spatial heterogeneity will be conducted to address how the upscaled NDVI varies according to the aggregated pixel scales in general cases.

\section{ACKNOWLEDGEMENTS}

This research was supported by the Innovation Seed Fund for Horticulture Development grant from the University of Melbourne and Department of Economic Development, Jobs, Transport and Resources, Victoria. The author is gratefully thanks to Lisa Kimmorley for the access to Curly Flat Vineyard. And also thanks to Andrew P. Nolan with the help of UAV dataset collection in the field.

\section{REFERENCES}

Abuzar, M., Sheffield, K., Whitfield, D., O’Connell, M., McAllister, A. (2014). Comparing inter-sensor NDVI for the analysis of horticulture crops in south-eastern Australia. Int. Arch. American Journal of Remote Sensing, 2(1), 1-9.

Grenzdorffer, G. J., Engel, A., Teichert, B. (2008). The photogrammertric potential of low-cost UAVs in forestry and agriculture. The International Archives of the Photogrammetry, Remote Sensing andSpatial Information Sciences, 31(B3), 1207-1214.

Jones, H. G., Vaughan, R. A. (2010). Remote sensing of vegetation: Principles, techniques, and applications. Oxford university press.

Silleos, N. G., Alexandridis, T. K., Gitas, I. Z., Perakis, K. (2006). Vegetation Indices: Advances Made in Biomass Estimation and Vegetation Monitoring in the Last 30 Years, Geocarto International. 21(4), 2128.

Solano, R., Didan, K., Jacobson, A., Huete, A. (2010). MODIS Vegetation Indices (MOD13) C5 User's Guide, Version, 2, 2010.

Weier, J., Herring, D. (2009). Measuring Vegetation (NDVI \& EVI), Earth Observatory. 